\title{
Three-stage method for intentional controlled islanding of power systems
}

\author{
Shaoxiang $\mathrm{XU}^{1}$, Shihong MIAO${ }^{1}$
}

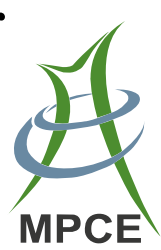

\begin{abstract}
Power system controlled islanding is an emergency control to stop the propagation of disturbances and to avoid blackouts. This paper presents a three-stage method for intentional controlled islanding of power systems. It enables to search out reasonable islanding cutsets, which have the minimum load-generation imbalance or the minimal power flow disruption, without low-voltage problems. In the first stage, a self-adaptive graph simplification algorithm is proposed to obtain a two-terminal graph as a suitable islanding cutset search area from the original power network graph model. In the second stage, an islanding cutset search algorithm is designed to find all of islanding cutsets, including the minimum load-generation imbalance cutset, in the two-terminal graph. In the third stage, an islanding scheme checking algorithm is developed to examine the outputs of stage two. It uses the depth first search algorithm to determine reasonable islanding cutsets without low-voltage problems. The IEEE 30-bus system and the IEEE 118-bus system are utilized to demonstrate the proposed method. The simulation results show its validity and accuracy in large-scale power systems.
\end{abstract}

CrossCheck date: 17 September 2017

Received: 22 November 2016/Accepted: 17 September 2017/ Published online: 22 December 2017

(C) The Author(s) 2017. This article is an open access publication

$\triangle$ Shihong MIAO

shmiao@mail.hust.edu.cn

Shaoxiang XU

Xushaoxiang@outlook.com

1 State Key Laboratory of Advanced Electromagnetic Engineering and Technology, Hubei Electric Power Security and High Efficiency Key Laboratory, School of Electrical and Electronic Engineering, Huazhong University of Science and Technology, 1037 Luoyu Road, Wuhan 430074, China
Keywords Intentional controlled islanding, Islanding cutset, Power flow tracing, Minimal power imbalance, Minimal power flow disruption

\section{Introduction}

Since 1990, the modern electricity market has expanded in several countries. A great number of power systems operate close to their limits due to severe economic stresses. Moreover, power systems face a variety of threats including component failures, human errors and natural disasters. These internal and external threats may cause the loss of synchronism, cascading outages or even the complete blackout. Studies of historic blackouts or outages reveal that a feasible and reliable real-time power defense system, which has been envisioned by Brazil [1], France [2], and the U.S. [3], needs to be developed. A controlled islanding strategy is an important part of the modern power defense system. It eliminates severe disturbances and oscillations by splitting the power system into a group of subsystems or so-called islands under different system operating conditions and network configurations. It acts as the last step to prevent the entire power system from blacking out in case of the loss of synchronism.

Most approaches to islanding aim to identify, as primary objective, stable islands with the minimal load shedding. To find reasonable islanding cutsets, all subsystems must satisfy some constraints, such as generator coherency, loadgeneration imbalance/power flow disruption, transmission line thermal limits, voltage limits, transient stability, etc. Among the aforementioned constraints, generator coherency and load-generation imbalance/power flow disruption are two basic constraints for the selection of islanding cutsets. The definition of generator coherency constraint is 
that generators are divided into coherent groups and each island contains only one group of coherent generators. Hence, power system oscillations can be eliminated by satisfying this constraint. The load-generation imbalance/ power flow disruption should be as small as possible so that subsystem disturbances are restricted in allowable limits.

Several controlled islanding methods have been proposed to search for islanding cutsets by satisfying two or more constraints. References [4-6] present a two-phase strategy to split power systems using ordered binary decision diagrams (OBDDs). In these methods, the network simplification is based on geographic areas or control areas that only cross-regional transmission lines would be tripped after the power system lost its synchronization. It will lead to a large load-generation imbalance in each island if the simplified network is used to split the power system. References [7, 8] use graph partitioning software METIS and pMETIS, based on multilevel k-way schemes, to find stable islands. The number of islands given by these two programs may be more than desired, for a group of generators are divided into two or more islands. Tripping unnecessary transmission lines leads to a longer resynchronization time, and is not beneficial to subsystem stability. In [9, 10], a brute force method is applied to determine islanding cutsets in an interface network reduced from the original graph model. The scalar parameter, which regulates the scale of the interface network, is set by operators depending on their experience. Therefore, these methods are not self-adaptive islanding methods for different kinds of power systems. References [11, 12] develop mixed integer linear programming-based optimization methods for controlled islanding. Like [7, 8], a group of coherent machines may be assigned into more than one island after serious disturbances. References [13, 14] propose respectively a multilevel kernel k-means method and a spectral partitioning method. Both methods ignore the generator coherency constraint which is one of the most fundamental constraints in the islanding algorithm. A twostep spectral clustering controlled islanding algorithm is presented in [15]. It brings an improvement to [13] that the generator coherency constraint can be followed by spectral method. Reference [15] uses the eigenvalue and the eigenvector of a system state matrix derived from the linearized dynamic model of generators to identify coherent generators. Under each system condition, this method could only identify one type of coherent groups. However, more than one type of coherent groups may be formed during a disturbance, and this method will not work in these circumstances. Reference [16] describes a constrained spectral clustering approach which is computationally more efficient than [15]. The partition of the original power system in [16] is too coarse to detect the minimal power flow disruption. Reference [17] discusses a hierarchical spectral clustering method to split power systems by using DC power flow. Thus, this method is only effective on linearized systems. In [18], coherent generators and load buses are clustered by a modified k-means technique, which employs the distance between the load bus and the centroid of a coherent group. Sometimes, some buses may not be assigned to any island and this would cause a large load-generation imbalance. Reference [19] employs a power flow tracing method to determine the domain of each group of coherent generators, and it aims to split the network along the boundaries of groups that may miss some reasonable islanding cutsets. The power flow tracing method, which identifies the cutsets according to the predetermined threshold value, is proposed in [20]. It will not determine proper islands if the predetermined threshold value is set too high or too low by operators.

In this paper, a three-stage method for intentional islanding of power systems is proposed to avoid the above mentioned problems. The aim of this method is to find islanding cutsets with the minimal load-generation imbalance or the minimal power flow disruption for any given number of islands, while ensuring that each island follows the voltage limit constraint. There are three contributions of this method for intentional controlled islanding. In the first place, a self-adaptive graph simplification algorithm is proposed in stage one. This algorithm, which matches various operating conditions and network configurations, can automatically determine the islanding cutset search area in different power systems. Secondly, the improved recursive merge algorithm is designed to find out all islanding cutsets in the two-terminal graph. Thirdly, an islanding scheme checking algorithm is developed to export reasonable islanding cutsets. The depth first search algorithm is used to check islanding cutsets and prevent islands from the occurrence of low-voltage problems.

The organization of this paper is as follows: Sect. 2 provides the background of power flow tracing method, graph theory and recursive merge algorithm. Section 3 presents in detail the three-stage method and its procedure. In Sect. 4, the IEEE 30-bus and 118-bus test system are used to demonstrate its performance. Finally, conclusions and further research directions are drawn in Sect. 5.

\section{Background information}

\subsection{Graph theory preliminaries}

Graph models have been widely used to represent power system networks in intentional controlled islanding. In this paper, the power system network is converted into an undirected, connected, weighted graph with buses and lines 
represented as vertices and edges respectively. Each edge has two weights, the inflow weight and the outflow weight. Edge weights are equal to values of the real power at each terminal bus of the line. The graph model for a 5-bus system is shown in Fig. 1 where the nodal inflow is negative and the nodal outflow is positive. The load-generation imbalance (real power) for island 1 in Fig. 1 is:

$\Delta P=P_{23}+P_{25}+P_{45}=4$

And the power flow disruption (edge weight) for island 2 is:

$\Delta w=\left|w_{32}\right|+\left|w_{52}\right|+\left|w_{54}\right|=7$

An adjacency matrix $\boldsymbol{A}(\boldsymbol{G})=\left[a_{i j}\right]$, which is the mathematical model for graphs, indicates whether the two vertices in each pair are adjacent or not in the graph. It is a $(0,1)$-matrix with zeros on its diagonal. Elements $a_{i j}$ $(i \neq j)$ will be one if there is an edge from vertex $i$ to vertex $j$. If not, $a_{i j}$ will be zero. Figure 2 shows the adjacency matrix for an undirected 5 nodes graph.

\subsection{Power flow tracing method}

The power flow tracing method allocates the total cost of transmission service between all suppliers and consumers in the electric-power market. Reference [21] proposes a power flow tracing method based on the Kirchhoff's Current Law, which uses a topological methodology. The proportional sharing principle provides the basis of this method for determining the contribution of each generator to the load in each bus. The upstreamlooking algorithm, which allows the assessment of the real power output from a particular generator goes to a

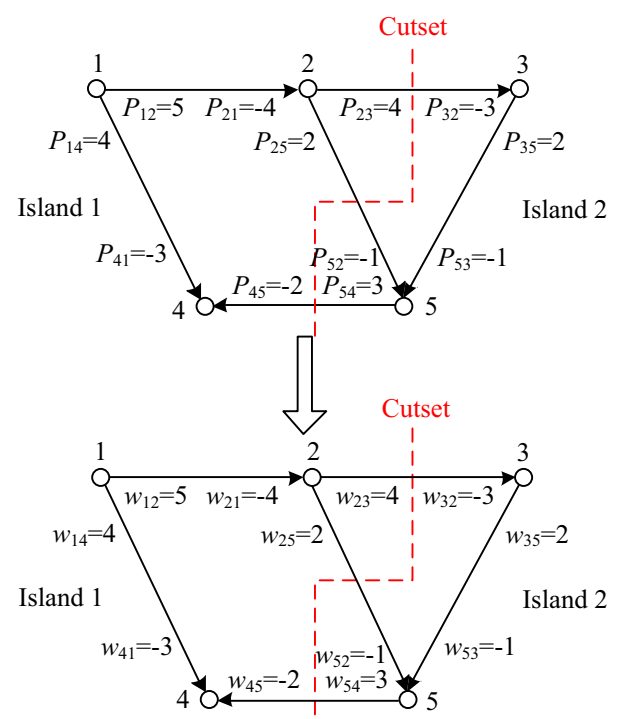

Fig. 1 Graph model for the 5-bus system

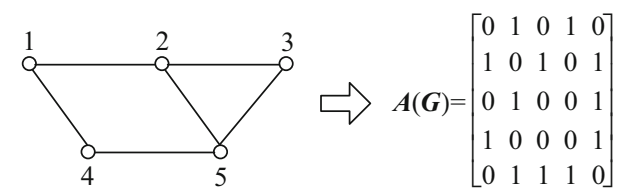

Fig. 2 Adjacency matrix for undirected 5 nodes graph

particular load, is used in this study. For an n-bus system, the element of the upstream matrix $\boldsymbol{A}_{u}$ is:

$\left[\boldsymbol{A}_{u}\right]_{i j}= \begin{cases}1 & i=j \\ -\left|P_{j i}\right| / P_{j} & j \in \alpha_{i}^{u} \\ 0 & \text { otherwise }\end{cases}$

where $P_{j i}$ is real power from bus $j$ to bus $i, P_{j}$ is total inflows at bus $j$. The relationship among these matrices is

$\boldsymbol{A}_{u} \boldsymbol{P}=\boldsymbol{P}_{G}$

where $\boldsymbol{P}$ is the matrix of total inflows at each bus, $\boldsymbol{P}_{G}$ is the matrix of generations at each bus. The load demand $P_{L i}$ can be derived from (2).

$P_{L i}=\frac{P_{L i}}{P_{i}} P_{i}=\frac{P_{L i}}{P_{i}} \sum_{k=1}^{n}\left[A_{u}^{-1}\right]_{i k} P_{G k} \quad i=1,2, \ldots, n$

where $\sum_{k=1}^{n}\left[\boldsymbol{A}_{u}^{-1}\right]_{i k} P_{G k}$ is the $i^{\text {th }}$ element of the inverse of (2). The contribution of the $k^{\text {th }}$ generator to the $i^{\text {th }}$ load demand is equal to:

$\eta_{i k}=P_{L i} P_{G k} \frac{\left[\boldsymbol{A}_{u}^{-1}\right]_{i k}}{P_{i}}$

\subsection{Recursive merge algorithm}

The recursive merge algorithm is present to enumerate all minimal cutsets of a two-terminal graph in [22, 23]. It begins the merging procedure from the source node and recursively merges adjacent nodes one by one into the source set. An example of the recursive merge algorithm is illustrated in Fig. 3, and the procedure for this algorithm is briefly described as follow:

1) Determine the first cutset, which is the set of all emitting edges from source node, for the original twoterminal graph.

2) Merge adjacent nodes to source set.

3) Send the new two-terminal graph's hash code to hash table.

4) If the graph's hash code is not in hash table, go to 5). Otherwise, the cutset of this graph has been searched, and this subgraph's recursive process is exited.

5) If there is not any redundant node in the subgraph, output the cutset and go to 6). Otherwise, merge redundant nodes into source set and go to 3 ). 


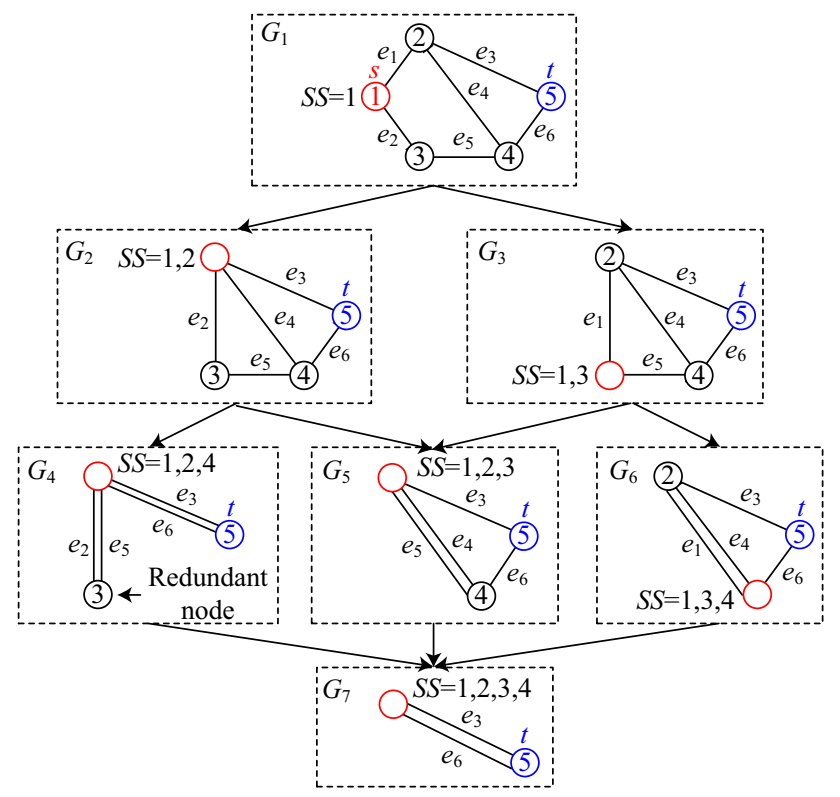

Fig. 3 Example of recursive merge algorithm

6) If there are only source set and sink node in the subgraph, output the last cutset and end the program. Otherwise, go to 2).

\subsection{Generator coherency}

Electro-mechanical oscillations, which can lead to a loss of synchronism, may be the consequence of a severe disturbance in the power system. To eliminate the power system oscillations, each group of coherent generators must be separated into different islands. Like references $[4-9,11,13,16,19,20]$, our method receives the information of coherent groups from the identification program, in order to search for appropriate islands which satisfy the generator coherency constraint and other constraints.

\section{Three-stage method for controlled islanding}

\subsection{Stage one: locating the search area}

With the growing number of substations and transmission lines, the scale of power system is larger than ever. Reference [5] proves that the intentional controlled islanding problem is a NP-hard problem. It means that the computational burden will be significant if islanding cutsets are searched from an original graph model. Reference [19] indicates that the reasonable islanding cutsets are located within the range of a small area among asynchronous generator groups. Therefore, in order to reduce the computational burden, the first step is to reduce the size of the power system graph model.

The aim of this stage is to identify the islanding cutset search area and build a two-terminal graph model to find islanding cutsets in the next stage. At the beginning of this stage, power flow tracing method is used to determine how much real power at a load bus is supplied from each group of coherent generators. Then, the islanding cutset search area is located, depending on the proportion of inflows. There are two types of relationship between a load bus and a group of coherent generators: the strong connection and the weak connection. The strong connection means that the contribution of a group of coherent generators to a load bus is above the threshold value $\eta_{0}$. And, it will be a weak connection if the contribution is below the threshold value $\eta_{0}$. It is impossible for buses to have a strong connection with any group of coherent generators in the islanding cutset search area. Thus, a two-terminal graph, which acts as the simplification of the original graph, is constructed by using graph theory. In the two-terminal graph, terminal vertices are formed by load buses which have a strong connection with a group of coherent generators. Other compositions are represented by the islanding cutset search area. The direct two-terminal graph is applied to the constraint of the minimal load-generation imbalance in the next stage. And the undirected two-terminal graph is implemented on the constraint of the minimal power flow disruption to locate islanding cutsets.

The threshold value $\eta_{0}$, which controls the range of the islanding cutset search area, is the key point of simplification of power system graph model. It is possible that coherent generators cannot connect to each other when the threshold value is too small. And a certain amount of acceptable islanding cutsets will be missed if the threshold value is too large. In this study, a self-adaptive threshold is proposed to distinguish the type of each load bus. The selfadaptive threshold value can be calculated using the following steps:

1) Input results of real power flow tracing and coherent generators.

2) Rank buses by contributions of a group of coherent generators to load buses.

3) Select the first bus and save it into the first list.

4) If generators connect to each other via elements in the list/lists, go to 12). Otherwise, continue.

5) Select the next bus.

6) If it connects to any other buses in the list/lists, continue. Otherwise, go to 11).

7) It belongs to the corresponding list/lists.

8) If there is only one list, go to 4). Otherwise, continue.

9) If they have an intersection, continue. Otherwise, go to 5 ). 
10) Combine these lists, go to 8).

11) Create a new list and save it into the new list, go to 5).

12) The minimal contribution $\eta$ is the threshold value $\eta_{0}$.

The flow chart of this algorithm is shown in Fig. 4.

\subsection{Stage two: searching islanding cutsets}

From the description in Sect. 2.3, it is observed that only the graph structure is taken into consideration in the recursive merge algorithm. Without taking into account the weighted model, the algorithm is not suitable for searching islanding cutsets of power systems.

In this section, the recursive merge algorithm is improved to solve this problem. A few steps, which are relevant to edge weights, have been inserted into this algorithm. Consequently, this improved algorithm, which is called islanding cutset search algorithm, can be used to search power system graph models for islands.

The minimal power disruption is taken as the objective function for the controlled islanding scheme. It is represented by:

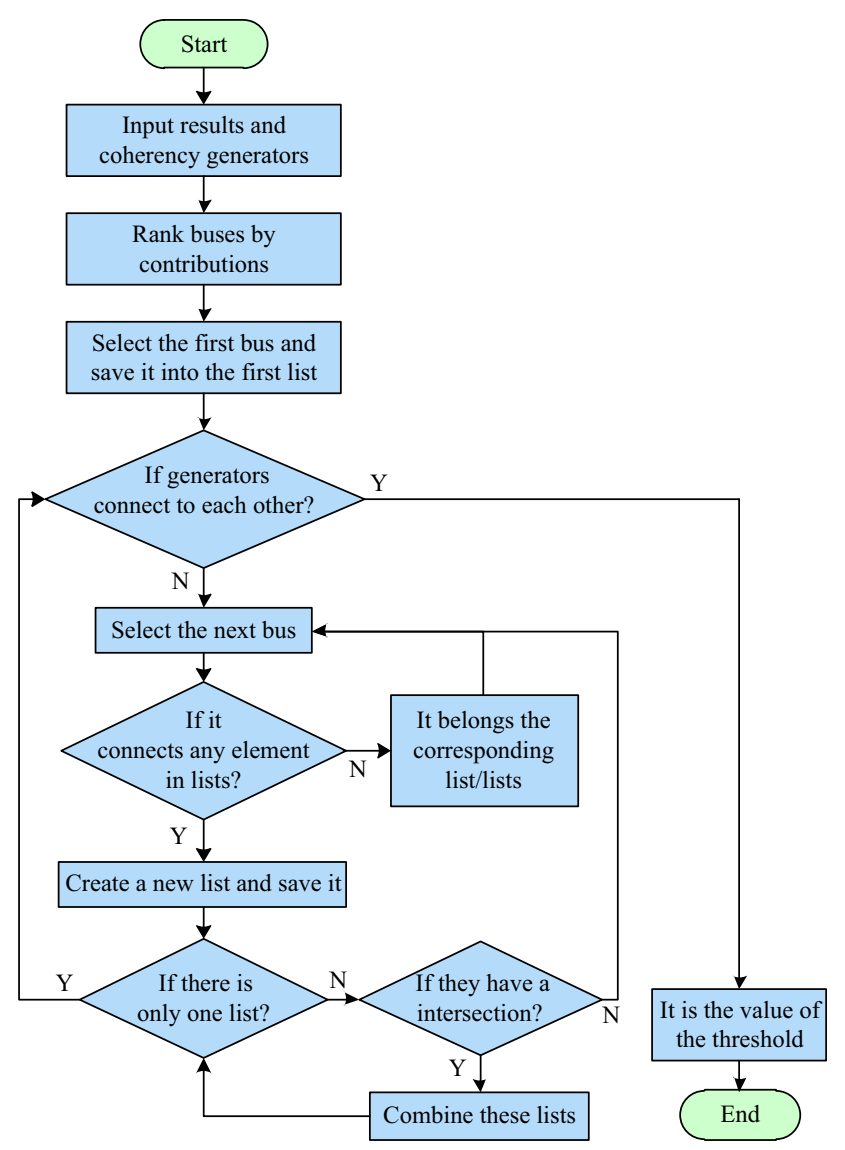

Fig. 4 Flow chart of procedure for self-adaptive threshold
$\Delta w_{d i s}=\min \left(\sum_{k \in S_{i}}\left|w_{k}\right|\right)$

where $w_{k}$ is the edge weight; $S$ is the set of all tripped lines in island $i$. The objective function aims to achieve the lowest sum of absolute edge weights which is the minimal power disruption cutset for the power system. Like (6), the minimal load-generation imbalance has a similar function. It can be expressed as:

$\Delta w_{i m}=\min \left(\left|\sum_{k \in S_{i}} w_{k}\right|\right)$

The object of this algorithm is to find the cutset which has the lowest absolute sum of edge weights.

The flow chart of this algorithm is given in Fig. 5. And the detailed procedure is as follows.

1) Input the $N$ dimensions adjacency matrix of original two-terminal graph and edge weights.

2) Select an adjacency matrix, send its hash code to hash table.

3) If its hash code already exists in the hash table, go to 8). Otherwise, continue.

4) If there is any redundant vertex in matrix, go to 8). Otherwise, continue.

5) Calculate the sum of edge weights which connect to source vertex.

6) If the sum of edge weight does not violate thresholds, continue. Otherwise, go to 8).

7) Output the islanding cutset.

8) If all matrices in this dimension are calculated, continue. Otherwise, go to 2).

9) If there is only source vertex and sink vertex in the graph, go to 11). Otherwise, continue.

10) Merge adjacent vertex to source vertex, and obtain all (N-1) dimensions adjacency matrices, go to 2).

11) All islanding cusets are found out and recursive processing is ended.

\subsection{Stage three: checking islanding schemes}

A number of islanding cutsets are found through the process of stage one and stage two. Bus voltages are within the normal range in strong connection areas because the graph simplification algorithm keeps the integrity of power networks in these areas. On the contrary, the low-voltage problem occasionally appears in the islanding cutset search area after power systems are split. The most convincing explanation is that some substations are connected in series without any power plant and electrical impedances cause a large voltage drop in long transmission lines. The characteristic of this kind of network structure is that there is at 


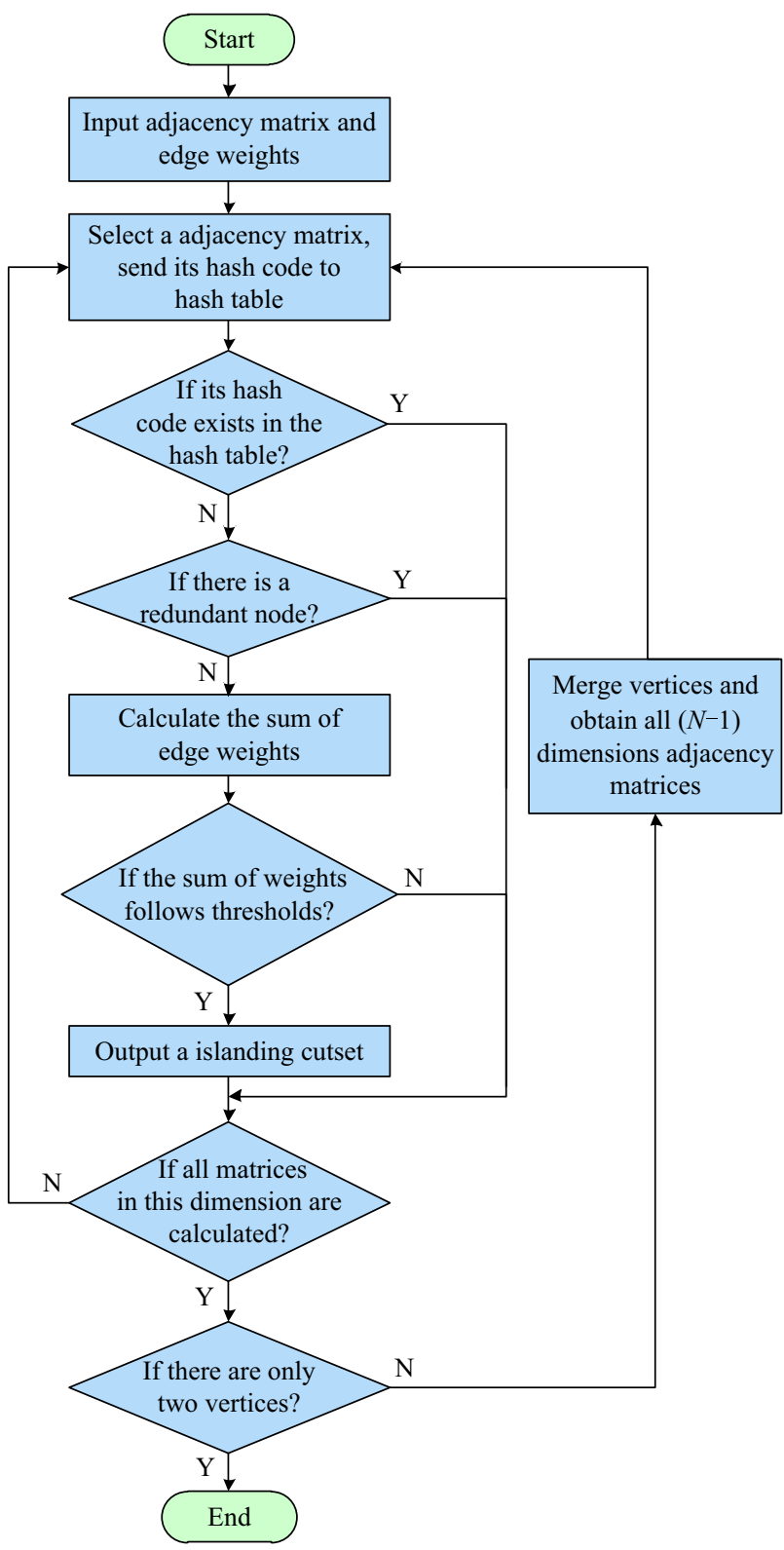

Fig. 5 Flow chart of islanding cutset search algorithm

least one cut-vertex in this part of network. An islanding scheme checking algorithm is developed to search the nearby area of the terminal bus for cut-vertices. Meanwhile, it also counts the number of buses in each path. The islanding cutset will be declined by the checking algorithm if the number of substations in series is larger than the maximum value. The depth first search algorithm acts as the core of the islanding scheme checking algorithm. The load bus which locates at the beginning or the end of the tripped transmission line is specified as a root node. The checking algorithm searches the graph model for nodes from the root to the $n^{\text {th }}$ generation. The islanding scheme will pass the checking process if more than one branch exists in $n^{\text {th }}$ generation. In addition, the island will be accepted if any PV bus is found by this algorithm in the model. To illustrate the checking algorithm, a graph model of a tripped transmission line and its nearby nodes are shown in Fig. 6. Assuming that the number of generation $n$ is 2 in this model, only one path $\{1 \rightarrow 2 \rightarrow 3 \rightarrow 4\}$, which connects to the root node, exists in Fig. 6a so that it is not a reasonable scheme for intentional controlled islanding. The reasonable island, which is shown in Fig. 6b, has two branches, $\{3 \rightarrow 4\}$ and $\{5 \rightarrow 7\}$, for the $2^{\text {nd }}$ generation.

\subsection{Procedure for the three stage method}

The procedure for three-stage method for intentional controlled islanding is presented in this section. In Fig. 7, the flow chart of three-stage method consists of four parts, input, locating, searching and checking. The function of each part is described as follows:

1) Input: Input the power network graph model, coherent generators, and branch weights.

2) Locating: Activate program. Import graph model and coherent generators. Derive the simplified two-terminal graph based on power flow tracing method and graph theory.

3) Searching: Import the two-terminal graph and branch weights. Search for islanding cutsets.

4) Checking: Import and check islanding schemes. Output all reasonable results.

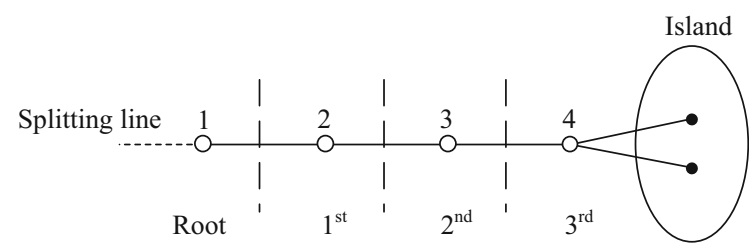

(a) Unreasonable island

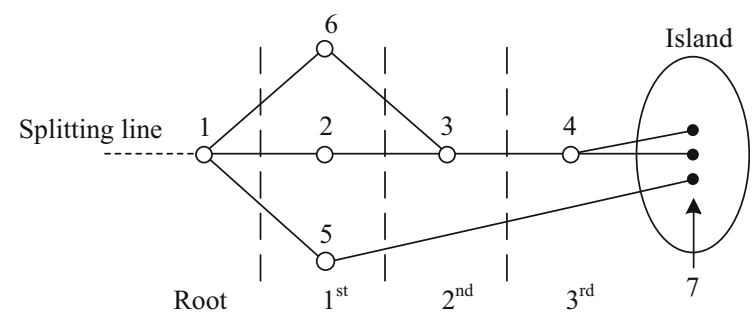

(b) Reasonable island

Fig. 6 Graph models for islanding power system 


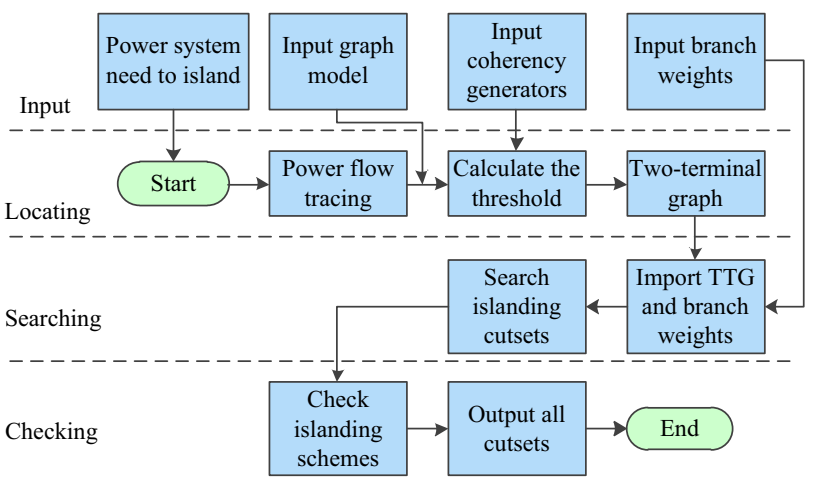

Fig. 7 Flow chart of three-stage method

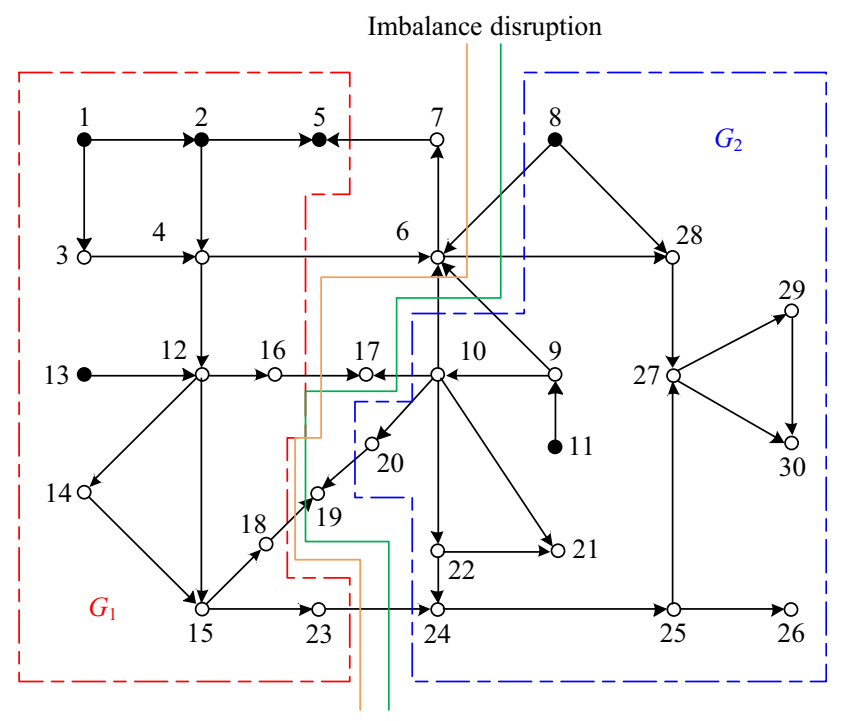

Fig. 8 IEEE 30-bus test system and its islanding cutsets

\section{Case studies}

The IEEE 30-bus and 118-bus test system are used to demonstrate the performance of the three-stage method in this section. The value of generation $n$ is 2 in the checking stage. The testing program is compiled by $\mathrm{C}++$ on a personal computer. And configurations of this $\mathrm{PC}$ are 2.66 GHz CPU and 4G RAM.

\subsection{IEEE 30-bus test system}

The IEEE 30-bus system is used to illustrate how the graph simplification algorithm works on the original graph model. The structure of this system is shown in Fig. 8. And the data of this system can be found in [24]. Generators are divided into two groups $G_{1}=\{1,2,5,13\}$ and $G_{2}=\{8,11\}$ after a severe disturbance [4].

The threshold value $\eta_{0}^{1}$ is 1 for the coherent group $G_{1}$ that all load bus in the strong connection area are entirely supplied by $G_{1}$. In the strong connection area of $G_{2}$, the generator 8 and the generator 11 will connect to each other via buses in the list if an appropriate threshold value is set. The step by step process of the threshold value calculation for the coherent group $G_{2}$ is listed on Table 1. The selfadaptive threshold value for coherent group $G_{2}$ is 0.6175 in this case. The generator 8 is connecting to the generator 11 when the bus 25 is allocated to the strong connection area. The bus 26 is specified as an element of the strong connection area due to the contribution of $G_{2}$ to the bus 26 is the same as to the bus 25. An undirected two-terminal graph is formed to represent the original graph model in Fig. 9. The cutset of the minimal load-generation imbalance is $\left\{l_{6-8}, l_{6-9}, l_{6-10}, l_{6-28}, l_{16-17}, l_{18-19}, l_{23-24}\right\}$. The loadgeneration imbalance is $-0.08 \mathrm{MW}$ in island $G_{1}$ and 0.02 MW in island $G_{2} .43 .42 \mathrm{MW}$ is the power flow disruption

Table 1 Process of the threshold value calculation for $G_{2}$

\begin{tabular}{lllll}
\hline Step No. & Bus & Contribution (\%) & Bus number list & Connectively (Y/N) \\
& No. & & & \\
\hline 1 & 9 & 100 & $\{9\}$ & $\mathrm{N}$ \\
2 & 10 & 95.78 & $\{9,10\}$ & $\mathrm{N}$ \\
3 & 20 & 95.78 & $\{9,10,20\}$ & $\mathrm{N}$ \\
4 & 21 & 95.78 & $\{9,10,20,21\}$ & $\mathrm{N}$ \\
5 & 22 & 95.78 & $\{9,10,20,21,22\}$ & $\mathrm{N}$ \\
6 & 28 & 70.03 & $\{9,10,20,21,22\}\{28\}$ & $\mathrm{N}$ \\
7 & 27 & 69.82 & $\{9,10,20,21,22\}\{27,28\}$ & $\mathrm{N}$ \\
8 & 29 & 69.82 & $\{9,10,20,21,22\}\{27,28,29\}$ & $\mathrm{N}$ \\
9 & 30 & 69.82 & $\{9,10,20,21,22\}\{27,28,29,30\}$ & $\mathrm{N}$ \\
10 & 24 & 61.75 & $\{9,10,20,21,22,24\}\{27,28,29,30\}$ & $\mathrm{N}$ \\
11 & 25 & 61.75 & $\{9,10,20,21,22,24,25,27,28,29,30\}$ & $\mathrm{Y}$ \\
12 & 26 & 61.75 & $\{9,10,20,21,22,24,25,26,27,28,29,30\}$ & $\mathrm{Y}$ \\
\hline
\end{tabular}




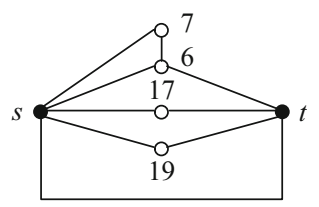

Fig. 9 Two-terminal graph for IEEE 30-bus test system

in island $G_{1}$ and $43.45 \mathrm{MW}$ is the power flow disruption in island $G_{2}$ for the cutset $=\left\{l_{6-8}, l_{6-9}, l_{6-10}, l_{6-28}, l_{10-17}, l_{18-19}\right.$, $\left.l_{23-24}\right\}$ which is the result of minimal power flow disruption for these islands. Strong connection areas and islanding cutsets are exhibited in Fig. 8.

\subsection{IEEE 118-bus test system}

The IEEE 118-bus test system is used to examine the proposed three-stage method and to compare the result with other algorithms. Two cases under different operating conditions are employed to demonstrate the accuracy for the minimal load-generation imbalance and the minimal power flow disruption.

\section{1) Test case 1}

The objective of this case is to find a cutset which minimize the load-generation imbalance. Generators are divided into three groups of coherent generators, $G_{1}=\{10,12,25,26,31\}, G_{2}=\{46,49,54,59,61,65$, $66,69\}$ and $G_{3}=\{80,87,89,100,103,111\}$ after several three-phase faults occurs at transmission lines [18] in this section. The data of the system can be found in Matpower, a power system analysis toolbox of MATLAB [25]. Like [18], the system is operated in optimal state where all constraints are satisfied and the fuel cost is minimized. All threshold values $\eta_{0}^{1}, \eta_{0}^{2}$ and $\eta_{0}^{3}$ are 1 for corresponding coherent groups in this case. Table 2 shows the load-generation imbalance for three methods and the comparison of results for four cutsets. The first and the second cutsets are produced by the proposed method which has less load-

Table 2 Results of the minimal load-generation imbalance

\begin{tabular}{|c|c|c|c|c|}
\hline \multirow[t]{2}{*}{ Cutset } & \multicolumn{3}{|c|}{$\sum P_{i j}(\mathrm{MW})$} & \multirow[t]{2}{*}{ Method } \\
\hline & Island1 & Island2 & Island3 & \\
\hline $\begin{array}{l}l_{15-33}, l_{35-36}, l_{34-37}, l_{34-43}, \\
\quad l_{30-38}, l_{24-70}, l_{24-72}, l_{75-77}, \\
\quad l_{76-77}, l_{69-77}, l_{68-81}\end{array}$ & -1.84 & 0.83 & 4.24 & \multirow[t]{2}{*}{$\begin{array}{l}\text { Three- } \\
\text { stage } \\
\text { method }\end{array}$} \\
\hline $\begin{array}{l}l_{15-33}, l_{34-36}, l_{34-37}, l_{43-44}, \\
\quad l_{30-38}, l_{24-70}, l_{24-72}, l_{75-77}, \\
\quad l_{76-77}, l_{69-77}, l_{68-81}\end{array}$ & 0.49 & -1.55 & 4.24 & \\
\hline $\begin{array}{l}l_{33-37}, l_{34-36}, l_{34-37}, l_{34-43}, \\
\quad l_{30-38}, l_{24-70}, l_{24-72}, l_{75-77}, \\
\quad l_{76-77}, l_{69-77}, l_{68-81}\end{array}$ & -4.48 & 3.5 & 4.24 & [19] \\
\hline
\end{tabular}

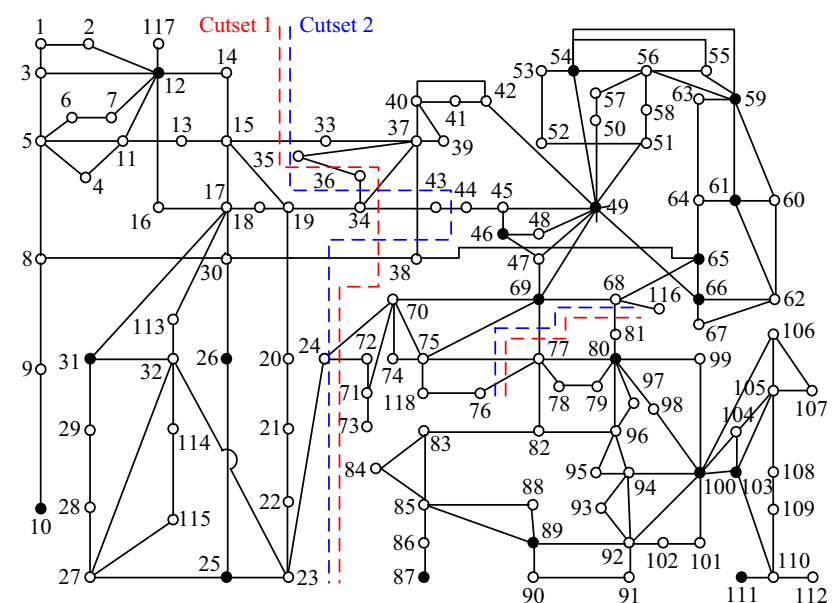

Fig. 10 Islanding cutsets for the minimal load-generation imbalance

generation imbalances than [19]. No low-voltage problem is appeared in solutions of power flow for these cutsets which are found by the three-stage method. The running time of this method is $0.049 \mathrm{~s}$, including $0.037 \mathrm{~s}$ in stageone, $0.010 \mathrm{~s}$ in stage-two and $0.003 \mathrm{~s}$ in stage-three, that it meets the computation speed requirement for on-line application. The first two islanding cutsets for the 118-bus system is displayed in Fig. 10.

2) Test case 2

The three-stage method is aimed at determining the minimal power flow disruption in this section. The IEEE

Table 3 Results of the minimal power flow disruption

\begin{tabular}{lccc}
\hline Cuset & & $\sum\left|P_{i j}\right|$ & Method \\
& & $(\mathrm{MW})$ & \\
\hline Cuset 1 & $l_{15-33}, l_{19-34}, l_{30-38}, l_{24-70}, l_{24-72}$ & 80.94 & Three-stage \\
& $l_{15-33}, l_{19-34}, l_{30-38}, l_{23-24}$ & 81.53 & method \\
& $l_{33-37}, l_{19-34}, l_{30-38}, l_{24-70}, l_{24-72}$ & 89.35 & \\
& $l_{15-33}, l_{19-34}, l_{30-38}, l_{23-24}$ & 81.53 & {$[15]$} \\
& $l_{15-33}, l_{19-34}, l_{30-38}, l_{24-70}, l_{24-72}$ & 80.94 & Brute force \\
& $l_{15-33}, l_{19-34}, l_{30-38}, l_{23-24}$ & 81.53 & method \\
& $l_{33-37}, l_{19-34}, l_{30-38}, l_{24-70}, l_{24-72}$ & 89.35 & \\
Cuset 2 & $l_{77-82}, l_{80-96}, l_{96-97}, l_{98-100}, l_{80-99}$ & 57.56 & Three-stage \\
& $l_{77-82}, l_{82-96}, l_{94-96}, l_{95-96}, l_{98-100}$, & 59.09 & method \\
& $l_{80-99}$ & & \\
& $l_{77-82}, l_{80-96}, l_{96-97}, l_{98-100}, l_{99-100}$ & 60.43 & \\
& $l_{77-82}, l_{80-96}, l_{96-97}, l_{98-100}, l_{80-99}$ & 57.56 & {$[15]$} \\
& $l_{77-82}, l_{80-96}, l_{96-97}, l_{98-100}, l_{80-99}$ & 57.56 & Brute force \\
& $l_{77-82}, l_{82-96}, l_{94-96}, l_{95-96}, l_{98-100}$, & 59.09 & method \\
& $l_{80-99}$ & & \\
& $l_{77-82}, l_{80-96}, l_{96-97}, l_{98-100}, l_{99-100}$ & 60.43 & \\
\hline
\end{tabular}


118-bus test system is under its normal state in this case. Generators form three groups, which are $G_{1}=\{10,12,25$, $26,31\}, G_{2}=\{46,49,54,59,61,65,66,69,80\}$ and $G_{3}=\{87,89,100,103,111\}$ after disturbance [15]. All threshold values $\eta_{0}^{1}, \eta_{0}^{2}$ and $\eta_{0}^{3}$ are 1 for corresponding coherent groups. Cutset 1 and Cutset 2, which are searched by these three methods, separate the whole system into three coherent groups. For both Cutset 1 and Cutset 2, the three cutsets with the smallest cut found by the brute force method are presented in Table 3, alongside which are the results returned by [15]. Additionally, the proposed method also lists three smallest cutsets for facilitating the comparison with other methods. The comparison in Table 3 shows that the three-stage method is more accurate than [15] and can find the cutset with minimal power flow disruption which is the same as the brute force method. No low-voltage problem exists in solutions of power flow for each island. Figure 11 shows the first two solutions for each cutset.

\section{Conclusion}

This paper proposes a self-adaptive three-stage method based on graph theory to determine intentional controlled islanding cutsets for power systems. This approach is applicable for various kinds of power systems under different system operating conditions and network configurations. It is also adjusted in accordance with different objectives of islanding, such as load-generation imbalance and power flow disruption. Meanwhile, each island is constrained by the generator coherency constraint as well as voltage limits constraints. Results of simulations

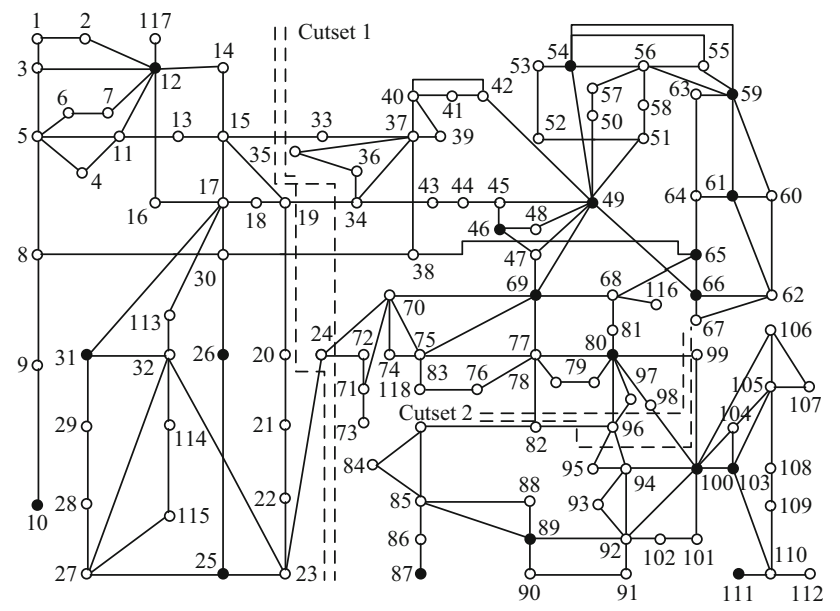

Fig. 11 Islanding cutsets for the minimal power flow disruption indicate that the three-stage method can be used in largescale power systems to find reasonable islanding cutsets, and it is more accurate than other methods.

Power system intentional controlled islanding is a very complicated problem. Emerging future research direction will focus on the transient and dynamic performance of this method. Moreover, pre- and post-islanding control, load and generation shedding strategies and a restoration scheme will be studied in the future.

Acknowledgements This work was supported by National Key Research and Development Program of China (2017YFB0903601) and National Natural Science Foundation of China (51777088).

Open Access This article is distributed under the terms of the Creative Commons Attribution 4.0 International License (http:// creativecommons.org/licenses/by/4.0/), which permits unrestricted use, distribution, and reproduction in any medium, provided you give appropriate credit to the original author(s) and the source, provide a link to the Creative Commons license, and indicate if changes were made.

\section{Appendix A}

The IEEE 118-bus test system is used to demonstrate the islanding scheme checking algorithm in this section. The power system is under its normal state. And the loadgeneration imbalance is taken into account in this case. Other assumptions are the same as Sect. 4.2. The first three minimal load-generation imbalance cutsets are listed in Table A1 and shown in Fig. A1.

The first two cutsets are rejected by the voltage checking algorithm. There are five substations connected in series in cutset $1 \quad(36 \rightarrow 34 \rightarrow 43 \rightarrow 44 \rightarrow 45)$ and cutset 2 $(19 \rightarrow 34 \rightarrow 43 \rightarrow 44 \rightarrow 45)$. AC power flows will not converge if cutset 1 and cutset 2 are used to split the power system. The cutset 3 is accepted by the checking algorithm so that it is the proper minimal load-generation imbalance cutset for this system.

Table A1 The first three minimal load-generation imbalance cutsets

\begin{tabular}{ll}
\hline Rank & Cutset \\
\hline 1 & $l_{80-99}, l_{98-100}, l_{77-82}, l_{82-96}, l_{96-95}, l_{96-94} ; l_{39-37}, l_{37-40}, l_{35-36}, l_{34-}$ \\
& $37, l_{19-34}, l_{38-65}, l_{24-70}, l_{71-72}$ \\
2 & $l_{80-99}, l_{98-100}, l_{77-82}, l_{82-96}, l_{96-95}, l_{96-94} ; l_{39-40}, l_{37-40}, l_{34-36}, l_{15-}$ \\
& $19, l_{18-19}, l_{19-20}, l_{34-37}, l_{38-65}, l_{24-72}, l_{24-70}$ \\
3 & $l_{80-99}, l_{98-100}, l_{77-82}, l_{82-96}, l_{96-95}, l_{96-94} ; l_{23-24}, l_{30-38}, l_{33-37}, l_{34-}$ \\
& $36, l_{34-37}, l_{34-43}$ \\
&
\end{tabular}




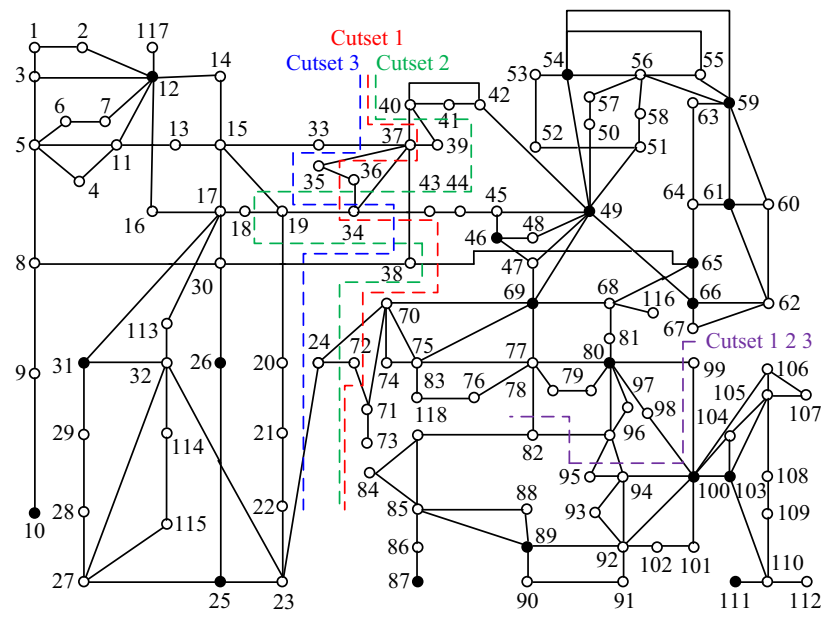

Fig. A1 The first three islanding cutsets for the minimal loadgeneration imbalance

\section{References}

[1] Filho VX, Pilotto LAS, Martins N et al (2001) Brazilian defense plan against extreme contingencies. In: Proceedings of the 2001 IEEE power engineering society summer meeting, Vancouver, Canada, 15-19 Jul 2001, pp 834-893

[2] Faucon O, Dousset L (1997) Coordinated defense plan protects against transient instabilities. IEEE Comput Appl Power 10(3):22-26

[3] Liu CC, Jung J, Heydt GT et al (2000) The strategic power infrastructure defense (SPID) system. A conceptual design. IEEE Control Syst Mag 20(4):40-52

[4] Sun K, Zheng D, Lu Q et al (2003) Splitting strategies for islanding operation of large-scale power systems using OBDDbased methods. IEEE Trans Power Syst 18(2):912-922

[5] Zhao Q, Sun K, Zheng D et al (2003) A study of system splitting strategies for island operation of power system: A two-phase method based on OBDDs. IEEE Trans Power Syst 18(4):1556-1565

[6] Sun K, Zheng D, Lu Q (2006) Searching for feasible splitting strategies of controlled system islanding. IEE Proc Gen Transm Distrib 153(1):89-98

[7] Xu G, Vittal V (2009) Slow coherency based cutset determination algorithm for large power systems. IEEE Trans Power Syst 25(2):877-884

[8] Li J, Liu CC, Schneider KP (2010) Controlled partitioning of a power network considering real and reactive power balance. IEEE Trans Smart Grid 1(3):261-269

[9] You H, Vittal V, Wang X (2004) Slow coherency based islanding. IEEE Trans Power Syst 19(1):483-491

[10] You H, Vittal V, Yang Z (2003) Self-healing in power systems: an approach using islanding and rate of frequency decline-based load shedding. IEEE Trans Power Syst 18(1):174-181

[11] Trodden PA, Bukhsh WA, Grothey A et al (2013) MILP formulation for controlled islanding of power networks. Int J Elect Power Energy Syst 45(1):501-508

[12] Trodden PA, Bukhsh WA, Grothey A et al (2014) Optimizationbased islanding of power networks using piecewise linear AC power flow. IEEE Trans Power Syst 29(3):1212-1220
[13] Peiravi A, Ildarabadi R (2009) A fast algorithm for intentional islanding of power systems using the multilevel kernel k-means approach. J Appl Sci 9(12):2247-2255

[14] Li H, Rosenwald GW, Jung J et al (2005) Strategic power infrastructure defense. Proc IEEE 93(5):918-933

[15] Ding L, Gonzalez-Longatt FM, Wall P et al (2013) Two-step spectral clustering controlled islanding algorithm. IEEE Trans Power Syst 28(1):75-84

[16] Quirós-Tortós J, Sánchez-García R, Brodzki J et al (2015) Constrained spectral clustering-based methodology for intentional controlled islanding of large-scale power systems. IET Gener Transm Distrib 9(1):31-42

[17] Sánchez-García RJ, Fennelly M, Norris S et al (2014) Hierarchical spectral clustering of power grids. IEEE Trans Power Syst 29(5):2229-2237

[18] Gomez O, Rios MA (2015) Real time identification of coherent groups for controlled islanding based on graph theory. IET Gener Transm Distrib 9(8):748-758

[19] Wang CG, Zhang BH, Hao ZG et al (2010) A novel real-time searching method for power system splitting boundary. IEEE Trans Power Syst 25(4):1902-1909

[20] Shao H (2015) Adaptive three-stage controlled islanding to prevent imminent wide-area blackouts. Dissertation, Durham University

[21] Bialek J (1996) Tracing the flow of electricity power. IEE Proc Gen Transm Distrib 143(4):313-320

[22] Lin H, Kuo S, The F (2003) Minimal cutset enumeration and network reliability evaluation by recursive merge and BDD. In: Proceedings of the IEEE $8^{\text {th }}$ symposium on computers and communication, Antalya, Turkey, 30 June-3 July 2003, pp 1341-1346

[23] Chang Y, Lin H, Kuo S (2004) Reliability evaluation of dependable distributed computing systems based on recursive merge and BDD. In: Proceedings of the IEEE $10^{\text {th }}$ pacific rim international symposium on dependable computing, Papeete, Tahiti, 3-5 Mar 2004, pp 197-206

[24] Kirschen D, Allan R, Strbac G (1997) Contributions of individual generators to loads and flows. IEEE Trans Power Syst 12(1):52-60

[25] Zimmerman RD, Murillo-Sánchez CE, Thomas RJ (2011) MATPOWER: steady-state operations, planning, and analysis tools for power systems research and education. IEEE Trans Power Syst 26(1):12-19

Shaoxiang XU received the B.E. degree in electrical engineering from the Wuhan University, Wuhan, China; the M.Sc. degree in electrical engineering from the Nanyang Technological University, Singapore. He is currently pursuing the Ph.D. degree at the Huazhong University of Science and Technology (HUST), Wuhan, China. His research interests include power system stability and control.

Shihong MIAO received the M.Sc. and Ph.D. degrees in electrical engineering from the Huazhong University of Science and Technology (HUST), Wuhan, China, respectively. Currently, he is a Professor at HUST. His research interests include power system protective relaying and control, micro-grid and renewable energy. 